\title{
Market behavior when preferences are generated by second order stochastic dominance
}

\author{
R.A. Dana* \\ August 2003
}

\begin{abstract}
We develop a theory of decision making and General Equilibrium for contingent markets when incomplete preferences are generated by second order stochastic dominance. Demand, Pareto-optima and Equilibria dominance are fully characterized. Demands and equilibrium allocations are non increasing functions of the pricing density and Pareto-optimal allocations are comonotone. They generalize mean-variance demands and CAPM equilibrium allocations which are non increasing affine functions of the pricing density. They are not observationaly distinguishable from those of von-NeumannMorgenstern decision makers with increasing strictly concave utilities nor from those of strict risk averse non-expected utility maximizers.

We also show that expenditure functions associated to second order stochastic dominance, provide microeconomic foundations for a class of law invariant risk-measures used in mathematical finance.
\end{abstract}

We are very grateful to the referee for his comments and suggestions. We have benefited from very stimulating conversations with E. Jouini and I. Meilijson and are particularly indebted to J. Werner for many fruitful remarks and suggestions and for his help editing the paper.

* Ceremade, Université Paris-Dauphine Place du maréchal Delattre de Tassigny, 75775 Paris cedex 16, France 


\section{Introduction}

Decision theorists model risk by a stochastic order. It would therefore be natural to use, in spite of their incomplete nature, a stochastic order rather than a utility function, in problems of microeconomic of uncertainty, finance or insurance. Although several papers have used a concept of demand or expenditure or efficiency for the order generated by second order stochastic dominance, without naming it, there has never been a systematic theory of individual decision making and General Equilibrium when incomplete preferences are generated by second order stochastic dominance (that we now on denote by S.S.D.). One can note that the papers that have used S.S.D. belong to a wide variety of literatures; efficiency pricing (Peleg-Yaari(1975), Chew and Zilcha (1990)), finance ( Ross-Dybvig (1982), Dybvig (1988), Kim (1994), Jouini-Kallal (2001), risk-sharing (Lansberger and Meilijson (1994), to name a few.

The first aim of this paper is to provide such a theory for contingent or complete markets. We shall first show that demand, expenditure, Paretooptima and equilibria under second order stochastic dominance may be fully characterized. We then show that they are not observationaly distinguishable from those of von-Neumann-Morgenstern decision makers with increasing strictly concave utilities nor from those of strict risk averse non-expected utility maximizers. They all have the same characteristics. The main tool of the analysis is a result due to Peleg-Yaari (1975) that any non-increasing function of the pricing density can be rationalized as the demand of some vNM EU maximizer with strictly increasing and strictly concave utility index. More precisely, let $p \in \mathbb{R}_{+}^{k}$ be a pricing density and let $w \in \mathbb{R}$ be a revenue. If $x$ is a non-increasing function of $p$ fulfilling $E(p x)=w$, then there exists a strictly increasing and strictly concave function $u$ such that $x$ solves

$$
\left\{\begin{array}{l}
\max E[u(c)] \text { s.t. } \\
E(p c) \leq E(p x)=w
\end{array}\right.
$$

While in Decision theory, risk is associated to a stochastic order, in finance and insurance, academics as well as practitionners have used measures of risk. Contrary to the stochastic order approach, measures of risk induce complete preferences on random variables. A standard measure of risk is value at risk. It 
has extensively been criticized by theorists. Other measures involving quantiles (or generalized inverse of distribution functions), have been proposed such as for example, law invariant coherent measures, premium principles (see Atzner et al (1999), Denneberg (1990), Föllmer and Schied (2002)). These measures are neither specifically based on microeconomic behavior nor related to equilibrium models.

Dybvig (1988) (an later Jouini-Kallal (2001) in the case of imperfections) defines the "utility price" $e(p, x)$ of a contingent claim $\mathrm{x}$ at pricing density $p$ as the minimal expenditure to get a claim that is at least as good as $x$ for any risk averse investor with v.N.M. increasing utility: $e(p, x)=$ $\left\{\begin{array}{l}\min E(p c) \text { s.t. } \\ E(u(c)) \geq E(u(x)) \forall u\end{array}\right.$. In the case of a uniform finite probability, Dybvig (1988) proves that $e(p, x)$ can be expressed in terms of $F_{p}^{-1}$ and $F_{x}^{-1}$ the quantile functions of $p$ and $x: e(p, x)=\int_{0}^{1} F_{p}^{-1}(1-t) F_{x}^{-1}(t) d t$. As the Choquet integral of a contingent claim with respect to a continuous convex distortion $f$ of a probability has value $E_{f}(x)=\int_{0}^{1} f^{\prime}(1-t) F_{x}^{-1}(t) d t$, one can see that $E_{f}(x)$ is the minimal expenditure to get a claim that is at least as good as $x$ for any price distributed as $f^{\prime}$ (if it exists). As these functionnals play a fundamental role in the theory of law invariant coherent measures, this provides a way of giving microeconomic foundations and potentially, an equilibrium analysis to a class of risk measures.

A second aim of the paper is therefore to use expenditure functions for S.S.D. to provide a class of law-invariant risk measures. We first generalize the formula giving $e(p, x)$ to any finite probability space. We deduce that a Choquet integral with respect to a continuous convex distortion $f$ of a probability is a minimal expenditure under S.S.D. if there exists a price distributed as $f^{\prime}$. Building on Dybvig (1988), we next compare $e(p, x)$ to a mean-variance criterion. We show that the utility price increases with its discounted expected value and decreases as the contingent claim becomes more disperse (the dispersion concept used is the order of dispersion introduced by Bickel-Lehmann). We show that efficient claims for S.S.D. are claims that maximize an analog of Sharpe ratio where standard deviation is replaced by a dispersion term. We then, endogenize the price system by introducing a concept of second order stochastic dominance equilibrium (denoted $\succsim_{2}$ equilibrium). It is interesting to compare S.S.D. equilibria to CAPM equilibria. In CAPM, agents' consumptions are affine increasing functions of aggregate endowment and the pricing density is an affine decreasing function of aggregate endowment while in a 
$\succsim_{2}$ equilibrium (equivalently in a v.N.M. equilibrium with increasing strictly concave utilities), agents' consumptions are non decreasing functions of aggregate endowment and aggregate endowment is a non decreasing function of the pricing density.

The paper is organised as follows: in section 2, we recall basic definitions used in the paper. In section 3, we define and characterize the concepts of second order stochastic dominance demand and strong efficiency. In section 4 , we define the utility price of a contingent claim, study its properties and relate it to the concept of law-invariant risk-measure. Sections five and six are devoted to Pareto optimality and equilibrium analysis.

\section{The model and a few basic definitions}

Given as primitive is a probability space $\left(\Omega, 2^{\Omega}, P\right)$. We assume that $\Omega$ is finite, $\Omega=\{1, \cdots, k\}$ and that $P=(\pi(1), \pi(2), \cdots, \pi(k))$. Contingent claims are identified to elements of $\mathbb{R}^{k}$. A contingent claim $x \in \mathbb{R}^{k}$ is denoted by $x=(x(1), \cdots, x(k))$. Two contingent claims $x$ and $y$ identically distributed are denoted $x \sim^{d} y$. Given a price $\tilde{p} \in \mathbb{R}^{k}$, we define the associated "pricing density" by $p=\left(\frac{\tilde{p}(1)}{\pi(1)}, \ldots, \frac{\tilde{p}(k)}{\pi(k)}\right)$. We therefore have $\tilde{p} \cdot x=E_{P}(p x)$ that we shall now on write $E(p x)$.

Let us first recall a few definitions. Let $F_{x}$ denote the distribution function of a contingent claim $x$ and $F_{x}^{-1}$ its generalised inverse:

$$
F_{x}^{-1}(t)=\inf \left\{z \in \mathbb{R} \mid F_{x}(z) \geq t\right\}
$$

\subsection{Second order stochastic dominance}

Definition $1 A$ contingent claim $x$ dominates $y$ in the sense of second order stochastic dominance (resp strictly dominates) denoted $x \succsim_{2} y$ (respectively $\left.x \succ_{2} y\right)$ if any of the following equivalent conditions are fulfilled:

1. $\int_{-\infty}^{t} F_{y}(s) d s \geq \int_{-\infty}^{t} F_{x}(s) d s, \forall t \in \mathbb{R}$ (resp. with a strict inequality for some $t$ ),

2. $\left.\left.\int_{0}^{t} F_{x}^{-1}(s) d s \geq \int_{0}^{t} F_{y}^{-1}(s) d s, \forall t \in\right] 0,1\right]$, (resp. with a strict inequality for some $t$ ), 
3. $E[u(x)] \geq E[u(y)], \forall u: \mathbb{R} \rightarrow \mathbb{R}$ concave increasing

(resp. with a strict inequality for some u concave increasing),

4. $y \sim^{d} x+\varepsilon$ for some $\varepsilon$ such that $E[\varepsilon \mid x] \leq 0$

(resp. where in addition $P(\varepsilon \neq 0)>0)$.

Two contingent claims $x$ and $y$ are second-order equivalent, denoted by $x \sim_{2} y$, if 1,2 or 3 holds with equality throughout. In other words, $x \sim_{2} y$ iff $x \sim^{d} y$.

It follows from assertion 4 and Jensen's inequality that $x \succ_{2} y$ iff for every $u: \mathbb{R} \rightarrow \mathbb{R}$ strictly concave increasing, $E[u(x)]>E[u(y)]$.

We shall extensively use this property.

\subsection{Comonotone functions}

Definition 2 1. Two random variables $x$ and $y$ are comonotone (resp anticomonotone) if $\left[x(s)-x\left(s^{\prime}\right)\right]\left[y(s)-y\left(s^{\prime}\right)\right] \geq 0$, for all $\left(s, s^{\prime}\right) \in \Omega^{2}$ (resp $\left[x(s)-x\left(s^{\prime}\right)\right]\left[y(s)-y\left(s^{\prime}\right)\right] \leq 0$, for all $\left.\left(s, s^{\prime}\right) \in \Omega^{2}\right)$.

2. A family of random variables $\left(x_{i}\right)_{i=1}^{n} \in \mathbb{R}^{k n}$ is comonotone if $\left[x_{i}(s)-x_{i}\left(s^{\prime}\right)\right]\left[x_{j}(s)-x_{j}\left(s^{\prime}\right)\right] \geq 0$, for all $i, j$ and for all $\left(s, s^{\prime}\right) \in \Omega^{2}$.

Remark: Let $f: \mathbb{R} \rightarrow \mathbb{R}$ and $g: \mathbb{R} \rightarrow \mathbb{R}$ be two non-decreasing functions. Then if $x=f(y)$ or $y=g(x)$, then $x$ and $y$ are comonotone but the converse does not necessarely hold. To be precise, two random variables $x$ and $y$ are comonotone iff for all $s \in \Omega,(x(s), y(s))$ belongs to the graph of a non decreasing correspondence.

An alternative characterization of comonotonicity (see Denneberg [1994]) expresses each $x_{i}$ as a non-decreasing, one-Lipschitz function of their sum:

Lemma 1 Denneberg's Lemma A family of of random variables $\left(x_{i}\right)_{i=1}^{n} \in$ $\mathbb{R}^{k n}$ is comonotone if and only if there exist continuous and non-decreasing functions $\left(f_{i}\right)_{i=1}^{n}\left(f_{i}: \mathbb{R} \rightarrow \mathbb{R}\right)$ with $\sum_{i=1}^{n} f_{i}=I d$, such that, for every $i$, $x_{i}=f_{i}\left(\sum_{j=1}^{n} x_{j}\right)$.

We shall also make use of the following results: 


\section{Lemma 2 1. Hardy-Littlewood's inequality}

$$
\int_{0}^{1} F_{x}^{-1}(1-t) F_{y}^{-1}(t) d t \leq E(x y) \leq \int_{0}^{1} F_{x}^{-1}(t) F_{y}^{-1}(t) d t
$$

2. If $x$ and $y$ are comonotone, then $\operatorname{cov}(x, y) \geq 0$.

3. If $x$ and $y$ are comonotone, then $F_{x+y}^{-1}=F_{x}^{-1}+F_{y}^{-1}$.

\section{Demand and Efficiency}

In this section, we define and characterize the demand correspondence for second order stochastic dominance. We show that the demand at pricing density $p$ is the set of non decreasing functions of $p$ which fulfill the budget constraint. We furthermore show that the demand correspondence for second order stochastic dominance is the union of the demand functions for strictly concave increasing expected utility maximisers. The proof is based on an argument originally made by Peleg and Yaari (1975) and further used by Jouini-Kallal (2001)

\subsection{Demand correspondence for second order stochastic dom- inance}

Let $(p, w) \in \mathbb{R}^{k+1}$ be a pricing-density income pair. The demand correspondence for second order stochastic dominance is defined by:

$$
\xi_{2}(p, w)=\left\{x \in \mathbb{R}^{k} \mid E(p x) \leq w \text { and } \exists x^{\prime} \succ_{2} x \text { with } E\left(p x^{\prime}\right) \leq w\right\}
$$

We start with a lemma:

Lemma 3 Let $x \in \mathbb{R}^{k}$ be such that $E(p x)=w$ and such that $x(\ell)<x(j)$ implies $p(\ell) \geq p(j)$ ( resp $p(\ell)>p(j)$ ). Then there exists a strictly increasing and concave (resp strictly concave) function $u$ such that $x$ solves $\left\{\begin{array}{l}\max E[u(c)) \text { s.t. } \\ E(p c) \leq E(p x)=w\end{array}\right.$

The proof, due to Peleg and Yaari [1975], is recalled in the appendix for completeness.

\section{Proposition 1}


1. If $\xi_{2}(p, w) \neq \emptyset$, then $p \gg 0$.

2. $x \in \xi_{2}(p, w)$ iff $E(p x)=w$ and $x$ is a non decreasing function of $p$.

The proof that $x \in \xi_{2}(p, w)$ implies that $E(p x)=w$ and $x$ is a non decreasing function of $p$ is due to Dybvig [1988]. The proof of the converse statement follows easily from Lemma 1. It may be found in the appendix.

\section{Corollary 1}

1. $x \in \xi_{2}(p, w)$ iff there exists $u: \mathbb{R} \rightarrow \mathbb{R}$ strictly concave increasing such that $x$ solves $\left\{\begin{array}{l}\max E u(c) \text { s.t. } \\ E(p c) \leq E(p x)=w\end{array}\right.$

2. $\xi_{2}(p, w) \neq \emptyset$ iff $p \gg 0$.

Proof. If $x \in \xi_{2}(p, w)$, the existence of $u$ follows from the proof of Proposition 1 . The converse statement follows from the definition of $\xi_{2}(p, w)$ and the caracterisation of strict second order stochastic dominance.

\subsection{Demand for $\succsim_{2}$ averse utility}

It follows from the previous section that the demand correspondence for second order stochastic dominance is the union of v.N.M. demands. This naturally raises the question of whether other families of utility may be considered. We define " $\succsim_{2}$ risk averse" utilities. These utilities have been used in various settings (for example, efficiency and insurance by Chew and Zilcha (1990)) and Gollier-Schlesinger(1996), Pareto-optimality and finance by Kim (1994)).

Let us first recall a definition.

Definition 3 A continuous utility $v: \mathbb{R}^{k} \rightarrow \mathbb{R}$ is " $\succsim_{2}$ risk averse" if $x \succsim_{2} y$ implies $v(x) \geq v(y)$ and strict risk averse denoted $\succ_{2}$ risk averse if $x \succ_{2} y$ implies $v(x)>v(y)$.

Examples and characterizations of risk averse utilities may be found in Chateauneuf et al(1997) and in Chew et al (1995). $\succsim_{2}$ risk aversion does not imply concavity or quasi-concavity of the utility function. 
Let $v: \mathbb{R}^{k} \rightarrow \mathbb{R}$ be a $\succ_{2}$ averse monotone increasing utility and let

$$
\xi_{v}(p, w)=\left\{\left.x \in \mathbb{R}^{k}\right|^{x=\arg } \begin{array}{l}
\max v(y) \text { s.t. } \\
E(p y) \leq w
\end{array}\right\}
$$

Since consumptions sets are unbounded, $\xi_{v}(p, w)$ may be empty, even in the case of v.N.M. utilities (see Bertzekas [1973] for example). Let us also define:

$$
\xi \mathcal{V}(p, w)=\left\{x \in \mathbb{R}^{k} \mid \exists v \succ_{2} \text { averse s.t. } x \in \xi_{v}(p, w)\right\}
$$

\section{Proposition 2}

$$
\xi_{\mathcal{V}}(p, w)=\xi_{2}(p, w)
$$

In particular, if $x \in \xi_{v}(p, w)$, then there exists $u: \mathbb{R} \rightarrow \mathbb{R}$ strictly concave increasing such that $x$ solves $\left\{\begin{array}{l}\max E u(c) \text { s.t. } \\ E(p c) \leq E(p x)=w\end{array}\right.$

Proof. The inclusion $\xi_{2}(p, w) \subset \xi_{\mathcal{V}}(p, w)$ follows from corollary 1. To prove that $\xi_{\mathcal{V}}(p, w) \subset \xi_{2}(p, w)$, one has to prove that, if $x \in \xi_{\mathcal{V}}$, then $E(p x)=w$ and $x$ is a non decreasing function of $p$. Assume that for some $(\ell, j), x(\ell)<x(j)$ and $p(\ell) \leq p(j)$. Then, as in the proof of Proposition 1, there exists $x^{\prime}$ such that $x^{\prime} \succ_{2} x$ and $E\left(p x^{\prime}\right) \leq w$, hence $v\left(x^{\prime}\right)>v(x)$, contradicting the definition of $x$. Hence $x(\ell)<x(j)$ implies $p(\ell)>p(j)$. Furthermore since $v$ is monotone increasing, $E(p x)=w$. The last assertion follows from corollary 1 .

\section{$3.3 \succ_{2}$ efficiency}

Let us next introduce the concept of strong efficiency which turn out to generalize the concept of mean-variance efficiency used in finance. We first recall the definition of Mean-Variance efficiency.

\subsubsection{Mean-Variance efficiency}

Let $\mathcal{W}$ denote the set of "mean -variance utilities" $W: \mathbb{R} \times \mathbb{R}_{+} \rightarrow \mathbb{R}$ strictly increasing in the first coordinate and strictly decreasing in the second.

A contingent claim $x$ is mean-variance efficient if there exists $W \in \mathcal{W}$ such that $x$ solves $\left\{\begin{array}{ll}\max & W(E(c), \operatorname{var}(c)) \text { subject to } \\ E(p c) \leq E(p x)\end{array}\right.$.

We recall that $x$ is mean-variance efficient iff there exists $a \in \mathbb{R}$ and $b \geq 0$ such that $x=a-b p$. In other words, $x$ is a decreasing affine function of $p$. 


\subsubsection{Strong efficiency}

In a similar way, we define the set of $\succ_{2}$ efficient claims.

$$
S E_{2}(p)=\left\{x \in \mathbb{R}^{k} \mid \exists u \uparrow \text { strictly concave s.t. } x \text { solves }\left\{\begin{array}{l}
\max E u(c) \\
E(p c) \leq E(p x)
\end{array}\right\}\right.
$$

It follows from Corollary 1 that

$$
S E_{2}(p)=\left\{x \in \mathbb{R}^{k} \mid \nexists x^{\prime} \succ_{2} x \text { with } E\left(p x^{\prime}\right) \leq E(p x)\right\}
$$

or equivalently that

$S E_{2}(p)=\left\{x \in \mathbb{R}^{k} \mid \nexists x^{\prime}\right.$ with $E\left(p x^{\prime}\right) \leq E(p x)$ and $E\left(u\left(x^{\prime}\right)\right)>E(u(x)), \forall u \uparrow$ s.c. $\}$

Define similarly

$$
S E_{\mathcal{V}}(p)=\left\{x \in \mathbb{R}^{k} \mid \exists v \underset{2}{\succ} \text { averse s.t. } x \text { solves }\left\{\begin{array}{l}
\max v(c) \\
E(p c) \leq E(p x)
\end{array}\right\}\right.
$$

It follows from proposition 2 that

$$
S E_{\mathcal{V}}(p)=S E_{2}(p)=\left\{x \in \mathbb{R}^{k} \mid x \text { is a non decreasing function of } p\right\}
$$

and hence that

$S E_{\mathcal{V}}(p)=\left\{x \in \mathbb{R}^{k} \mid \nexists x^{\prime}\right.$ with $E\left(p x^{\prime}\right) \leq E(p x)$ and $v\left(x^{\prime}\right)>v(x), \forall v \succ_{2}$ averse $\}$

A weaker concept of efficiency will be introduced in next section.

\section{Expenditure as a Measure of risk}

Theorists have often used quantile based measures of risk. The Choquet integral with respect to a convex distortion is an example of such measures. Following Dybvig's idea, we shall show that for some distortions, the Choquet integral may be obtained as an expenditure under second stochastic order, for a well-chosen price. 


\subsection{Definition of the Utility price}

Let $p \in \mathbb{R}^{k}$ be given. Consider the following problem $(\mathcal{E})$

$$
\operatorname{minimize}\left\{\begin{array}{l}
E(p c) \\
c \succsim_{2} x \\
c \in \mathbb{R}^{k}
\end{array}\right.
$$

The value function of $(\mathcal{E}), e(p, x)$ is called "Utility price" by Jouini-Kallal (2001). We keep their terminology. Let

$$
\psi(p, x)=\left\{\begin{array}{ll}
x^{\prime} \in \mathbb{R}^{k} \mid E\left(p x^{\prime}\right)=\min & E(p c) \\
& c \succsim_{2} x,
\end{array}\right\}
$$

be the set of minimizers.

A pricing-density is "revealing" if it is an injective function of the state of the world, equivalently if $\ell \neq j$ implies $p(\ell) \neq p(j)$.

The following properties may easily be proven:

Proposition 3 If $\psi(p, x) \neq \emptyset$, then

1. $p \geq 0$.

2. If $x^{\prime} \in \psi(p, x)$, then $x^{\prime}$ is anti-comonotone with $p$,

3. If $p$ is revealing, then if $x^{\prime} \in \psi(p, x), x^{\prime}$ is a function of $p$,

4. $e(\cdot, x)$ is positively homogeneous of degree 1 , concave and increasing. If $p \nsupseteq 0$, then $e(\cdot, x)=-\infty$.

\section{Proof.}

1. As in Proposition 1, assume that $p(\ell)<0$ for some $\ell$ and that $\psi(p, x) \neq \emptyset$. Let $x^{\prime}(j)=x(j), x^{\prime}(\ell)=x(\ell)+M, M>0$, then $x^{\prime} \succ_{2} x$ and $E\left(p x^{\prime}\right)<E(p x)$, a contradiction.

2. If $\psi(p, x) \neq \emptyset$, by the same proof as Proposition 1 , assertion $2, x^{\prime}(j)<x^{\prime}(\ell)$ and $p(j)<p(\ell)$ are incompatible. Hence $x^{\prime}(j)<x^{\prime}(\ell)$ implies $p(j) \geq p(\ell)$.

3 . If $p$ is revealing, by assertion $2, x^{\prime}(j)<x^{\prime}(\ell)$ implies $p(j)>p(\ell)$. Hence $x^{\prime}$ is a function of $p$. 
The proof of the last assertion is obvious.

We now generalize a result proven by Dybvig (1988) in the case of uniform probability:

Proposition 4 If $p \geq 0, e(p, x)=\int_{0}^{1} F_{p}^{-1}(1-t) F_{x}^{-1}(t) d t$ and $\psi(p, x) \neq \emptyset$ if and only if $p \geq 0$.

The proof may be found in the appendix.

Corollary 2 1) If $p$ is revealing, then $\psi(p, x)$ is a singleton.

2) If $p$ is not revealing, then $\psi(p, x)$ contains a unique function of $p$.

\subsection{Properties of the Utility price}

\subsubsection{Capacities}

We recall that a capacity on $\left(\Omega, 2^{\Omega}\right)$ is a set function $C: 2^{\Omega} \rightarrow \mathbb{R}$ such that $C(\emptyset)=0, C(\Omega)=1$, and for all $A, B \in 2^{\Omega}, A \subset B$, implies $C(A) \leq C(B)$. A capacity $C$ is convex if for all $A, B \in 2^{\Omega}, C(A \cup B)+C(A \cap B) \geq C(A)+C(B)$. Let $x: \Omega \rightarrow \mathbb{R}$ be a random variable. The Choquet integral of $x$ with respect to $C$ is defined by:

$$
E_{C}(x)=\int_{-\infty}^{0}(C(x \geq t)-1)+\int_{0}^{\infty} C(x \geq t) d t
$$

Let $f:[0,1] \rightarrow[0,1]$ non decreasing satisfy $f(0)=0, f(1)=1$ and $P$ be a probability on $\left(\Omega, 2^{\Omega}\right)$. Then $C=f(P)$ is a capacity. Moreover $f(P)$ is a convex capacity iff $f$ is convex. If $f$ is absolutely continuous, then

$$
E_{f}(x)=\int_{0}^{1} f^{\prime}(1-t) F_{x}^{-1}(t) d t
$$

\subsubsection{Dispersion}

The next two definitions related to the concept of dispersion of a random variable are due to Bickel-Lehmann (1977). 
Definition $4 A$ contingent claim $x \in \mathbb{R}^{k}$ dominates $y \in \mathbb{R}^{k}$ for BickelLehmann order (or $x$ is less dispersed than $y$ ) denoted $\underset{B L}{\underset{B L}{\succcurlyeq} y}$ if

$$
F_{y}^{-1}(q)-F_{y}^{-1}(p) \geq F_{x}^{-1}(q)-F_{x}^{-1}(p), 0<p<q<1
$$

Equivalently the map $p \rightarrow\left(F_{x}^{-1}(p)-F_{y}^{-1}(p)\right)$ is non increasing on $(0,1)$.

Definition 5 A map $\Delta: \mathbb{R}^{k} \rightarrow \mathbb{R}_{+}$is a measure of spread if it satisfies

1. $\Delta(a x)=|a| \Delta(x)$, for all $x \in \mathbb{R}^{k}$, for all $a \in \mathbb{R}$,

2. $\Delta(x+b)=\Delta(x)$, for all $b \in \mathbb{R}$

3. $\Delta(x)=\Delta(-x)$, for all $x \in \mathbb{R}^{k}$,

4. $\Delta(x) \leq \Delta(y)$ if $x \underset{B L}{\succsim} y$.

Examples of measures of spread are standard deviation and $\Delta(x)=\left[F_{x}^{-1}(t)-\right.$ $\left.F_{x}^{-1}(1-t)\right]$ for $t>\frac{1}{2}$ and more generally $\left.\Delta(x)=\left(\int_{\frac{1}{2}}^{1}\left[F_{x}^{-1}(t)-F_{x}^{-1}(1-t)\right)\right]^{\gamma} d \Lambda(t)\right)^{\frac{1}{\gamma}}$ where $\Lambda$ is a finite measure on $\left(\frac{1}{2}, 1\right)$.

\subsubsection{Properties of the Utility price}

We now elaborate on Dybvig's idea by introducing the dispersion order.

\section{Proposition 5}

1. Let $r$ be defined by $e(p, 1)=E(p)=\frac{1}{1+r}$. Then if $p \geq 0,(1+r) e(p, x)$ is a Choquet integral. More precisely $(1+r) e(p, x)=\int x d \nu$ where $\nu$ is a convex distortion of $P, \nu=\phi_{p} P$.

2. Let $D(p, x)=\frac{E(x)}{(1+r)}-e(p, x)$. Then $D(p, x) \geq 0, \forall x$ and if $x \underset{B L}{\succsim} y$, then $D(p, x) \leq D(p, y)$. Hence $e(p, x)=\frac{E(x)}{1+r}-D(p, x)$.

3. Let $\chi(p, x)=(D(p, x)+D(p,-x))=-(e(p, x)+e(p,-x))$. Then $\chi(p, x)$ is a convex comonotone measure of dispersion of $x$. 
Remarks: 1 . The distortion $\phi_{p}$ is piecewise linear since its derivative which is proportionnal to $F_{p}^{-1}$ is a step function.

2. $\phi_{p}$ is called the Lorenz curve of $p$ in the theory of measurement of inequality.

3. For $p \geq 0,-(1+r) e(p, x)$ is a comonotone coherent measure of risk (see Atzner et al (1999) and Delbaen (2000) for the definition and comments).

\subsubsection{An example: The tail conditionnal expectation}

Assume that $p=1_{A}$, then $\frac{1}{(1+r)}=P(A)$ and

$$
(1+r) e\left(1_{A}, x\right)=\frac{\int_{0}^{P(A)} F_{x}^{-1}(t) d t}{P(A)}=E\left[x \mid x \leq F_{x}^{-1}(P(A))\right]
$$

Hence $(1+r) e\left(1_{A}, x\right)$ is the tail conditionnal expectation. The corresponding measure of dispersion is :

$$
\chi\left(1_{A}, x\right)=\int_{0}^{P(A)}\left(F_{x}^{-1}(1-t)-F_{x}^{-1}(t)\right) d t=\int_{1-P(A)}^{1}\left(F_{x}^{-1}(t)-F_{x}^{-1}(1-t)\right) d t
$$

which is one of the example of measure of dispersion that we gave, with $\gamma=1$.

\subsection{Structure of the minimizers}

\subsubsection{The case of uniform probability}

As Dybvig (1988) and Jouini-Kallal (2001), let us first assume that the probability is uniform. The following results are essentially due to Jouini-Kallal (2001).

\section{Proposition 6}

1. $\psi(p, x)$ equals the convex hull of consumptions $x^{\prime}$ that fulfills $x^{\prime} \sim_{2} x$ and $x^{\prime}$ is anti-comonotone with $p$.

2. If $p$ is revealing, then $\psi(p, x)$ is a singleton: it is the unique nonincreasing function of $p$ distributed as $x$.

3. $e(p, x)=\max _{u} \min _{c}\left\{\begin{array}{l}E(p c) \\ E u(c) \geq E u(x)\end{array}\right.$ 


\subsubsection{The general case}

Proposition 7 Let $a_{i}>0$ for all $i$ and $b_{i}$ be such that

$$
F_{p}^{-1}(1-t)=\sum_{i=1}^{k} a_{i} 1_{\left[0, b_{i}\right]}(t)
$$

Then $x^{\prime} \in \psi(p, x)$ if and only if

$$
\int_{b_{i}}^{b_{i+1}} F_{x^{\prime}}^{-1}(t) d t=\int_{b_{i}}^{b_{i+1}} F_{x}^{-1}(t) d t, \text { for all } i
$$

The proof that follows from that of Proposition 4 is omitted.

\subsection{Weakly-efficient claims}

Following Dybvig (1988) and Jouini-Kallal (2001), we define efficient claims as claims for which there is no other less costly contingent claim at least as good for every risk averse investor with v.N.M. increasing utility. More precisely:

Definition 6 A contingent claim $x$ is $\succsim_{2}$ weakly-efficient at price $p$ iff there exists $u: \mathbb{R} \rightarrow \mathbb{R}$ increasing and concave such that $x$ solves $\left\{\begin{array}{l}\max E(u(c)) \text { subject to } \\ E(p c) \leq E(p x)\end{array}\right.$

Proposition 8 1. A contingent claim $x$ is weakly-efficient at price $p$ iff $E(p x)=\min _{\left\{c \succeq_{2} x\right\}} E(p c)$. Equivalently iff $E(p x)=e(p, x)$.

2. A contingent claim $x$ is weakly-efficient at price $p$ iff $x$ and $p$ are anticomonotone.

3. A contingent claim $x$ is weakly-efficient at price $p$ iff there exists $v$ : $\mathbb{R}^{k} \rightarrow \mathbb{R}$ increasing and $\succsim_{2}$ averse such that $x$ solves $\left\{\begin{array}{l}\max v(c) \text { subject to } \\ E(p c) \leq E(p x)\end{array}\right.$

4. A contingent claim $x$ with $E(p x)>0$ is efficient at price $p$ iff it maximizes

$$
\frac{E(R(x))-(1+r)}{D(p, R(x))}
$$

where $R(x)=\frac{x}{E(p x)}$ is the return of the contingent claim. 
Hence a contingent claim with positive price is efficient iff an analog of Sharpe ratio is maximized. The maximal value is $(1+r)$.

\section{$5 \quad$ Pareto optimality}

\subsection{Definitions}

We now consider an $n$ agents economy with initial endowments $\left(w_{i}\right)_{i=1}^{n} \in \mathbb{R}^{k n}$. Let $w=\sum_{i=1}^{n} \omega_{i}$ denote aggregate endowment.

We first give three definitions.

Definition 7 An allocation $\left(\tilde{x}_{i}\right)_{i=1}^{n} \in \mathbb{R}^{k n}$ is a $\succsim_{2}$ Pareto optimum, if there doesn't exist a feasible allocation $\left(x_{i}^{\prime}\right)_{i=1}^{n} \in \mathbb{R}^{k n}$ such that $x_{i}^{\prime} \succsim_{2} x_{i}$, for every $i$, with a strict inequality for some $i$.

Definition 8 A pair $\left[p^{*},\left(x_{i}^{*}\right)_{i=1}^{n}\right] \in \mathbb{R}^{k} \times \mathbb{R}^{k n}$ is $a \succsim_{2}$ equilibrium if

1. For every $i, E\left(p^{*} x_{i}^{*}\right) \leq E\left(p^{*} \omega_{i}\right)$ and there doesn't exist $x_{i} \succ_{2} x_{i}^{*}$, with $E\left(p^{*} x_{i}\right) \leq E\left(p^{*} \omega_{i}\right)$

2. $\sum_{i=1}^{n} x_{i}^{*}=\omega$.

Definition 9 A pair $\left[p^{*},\left(x_{i}^{*}\right)_{i=1}^{n}\right] \in \mathbb{R}^{k} \times \mathbb{R}^{k n}$ is $a \succsim_{2}$ equilibrium with transfer payments if

1. There doesn't exist $x_{i} \succ_{2} x_{i}^{*}$, with $E\left(p^{*} x_{i}\right) \leq E\left(p^{*} x_{i}^{*}\right)$

2. $\sum_{i=1}^{n} x_{i}^{*}=\omega$.

The following result may easily be proven:

Proposition 9 1. If there exists $\left(u_{i}\right)_{i=1}^{n}$ increasing strictly concave such that $\left(\bar{x}_{i}\right)_{i=1}^{n}$ is a Pareto optimum of the associated economy, then $\left(\bar{x}_{i}\right)_{i=1}^{n}$ is $a \succsim_{2}$ Pareto optimum.

2. If there exists $\left(u_{i}\right)_{i=1}^{n}$ increasing strictly concave such that $\left[p^{*},\left(x_{i}^{*}\right)_{i=1}^{n}\right] \in$ $\mathbb{R}^{k} \times \mathbb{R}^{k n}$ is an equilibrium (resp an equilibrium with transfer payments) of the associated economy, then $\left[p^{*},\left(x_{i}^{*}\right)_{i=1}^{n}\right] \in \mathbb{R}^{k} \times \mathbb{R}^{k n}$ is $a \succsim_{2}$ equilibrium (resp $a \succsim_{2}$ equilibrium with transfer payments). 
We shall prove that any $\succsim_{2}$ equilibrium (resp any $\succsim_{2}$ Pareto optimum) is an equilibrium (resp a Pareto optimum) of a strictly concave v.N.M. economy. The main tool of the proof is Peleg and Yaari's lemma.

\subsection{Caracterisations of $\succsim_{2}$ Pareto optimality}

Proposition 10 For a feasible allocation $\left(\bar{x}_{i}\right)_{i=1}^{n}$, the following conditions are equivalent

1. $\left(\bar{x}_{i}\right)_{i=1}^{n}$ is a $\succsim_{2}$ Pareto optimum.

2. The family of random variables $\left(\bar{x}_{i}\right)_{i=1}^{n}$ is comonotone.

3. There exists $\left(f_{i}\right)_{i=1}^{n}, f_{i}: \mathbb{R} \rightarrow \mathbb{R}$ continuous non decreasing with $\sum_{i=1}^{n} f_{i}=$ Id such that $x_{i}=f_{i}(\omega)$.

4. For every $p \gg 0$ such that $\omega(j)<\omega(\ell)$ implies $p(j)>p(\ell)$, there exists $\left(u_{i}\right)_{i=1}^{n}, u_{i}: \mathbb{R} \rightarrow \mathbb{R}$ strictly concave increasing such that $\left[p,\left(\bar{x}_{i}\right)_{i=1}^{n}\right]$ is an equilibrium with transfer payments of the corresponding v.N.M. economy.

5. There exists $\left(u_{i}\right)_{i=1}^{n}$ increasing strictly concave such that $\left(\bar{x}_{i}\right)_{i=1}^{n}$ is a Pareto optimum of the associated economy.

Proposition 11 For a feasible allocation $\left(\bar{x}_{i}\right)_{i=1}^{n}$, the following conditions are equivalent

1. $\left(\bar{x}_{i}\right)_{i=1}^{n}$ is a $\succsim_{2}$ Pareto optimum.

2. For any $p \gg 0$ such that $\omega(j)<\omega(\ell)$ implies $p(j)>p(\ell),\left[p,\left(\bar{x}_{i}\right)_{i=1}^{n}\right]$ is $a \succsim_{2}$ equilibrium with transfer payments.

The next proposition shows that no new qualitative behavior may be obtained by using risk averse utilities.

Proposition 12 For an allocation $\left(\bar{x}_{i}\right)_{i=1}^{n}$, the following conditions are equivalent

1. $\left(\bar{x}_{i}\right)_{i=1}^{n}$ is $a \succsim_{2}$ Pareto optimum. 
2. There exists $\left(v_{i}\right)_{i=1}^{n}, v_{i}: \mathbb{R}^{k} \rightarrow \mathbb{R}$ monotone increasing, $\succ_{2}$ averse such that $\left(\bar{x}_{i}\right)_{i=1}^{n}$ is a Pareto optimum of the associated economy.

\section{Proof.}

1. implies 2. It suffices to take $v_{i}(x)=E\left(u_{i}(x)\right)$ with $u_{i}$ constructed in Proposition 10, assertion 5 .

2. implies 1. since a Pareto optimum of a monotone increasing strictly strongly averse economy is clearly a $\succsim_{2}$ Pareto optimum.

We now give another characterisation of Pareto optima .

Proposition 13 An allocation $\left(\bar{x}_{i}\right)_{i=1}^{n}$ is $a \succsim_{2}$ Pareto optimum iff $\left(\bar{x}_{i}\right)_{i=1}^{n}$ is feasible and for some $p \gg 0$ revealing

i) $E(p \omega)=e(p, \omega)$ (aggregate endowment is efficient at price $p$ ),

ii) $e(p, \omega)=\sum_{i=1}^{n} e\left(p, \bar{x}_{i}\right)$, (the utility price of aggregate endowment is the sum of the utility prices of individual consumptions).

Proof. If

$$
e(p, \omega)=E(p \omega)=\sum_{i=1}^{n} E\left(p \bar{x}_{i}\right)=\sum_{i=1}^{n} e\left(p, \bar{x}_{i}\right)
$$

as $E\left(p \bar{x}_{i}\right) \geq e\left(p, \bar{x}_{i}\right)$, for every $i$, we have $E\left(p \bar{x}_{i}\right)=e\left(p, \bar{x}_{i}\right)$,. Hence, for every $i, \bar{x}_{i}(j)>\bar{x}_{i}(k)$ implies that $p(j)<p(k)$. Equivalently, for every $i, \bar{x}_{i}$ is a non increasing function of $p$ which implies the comonotony of $\left(\bar{x}_{i}\right)_{i=1}^{n}$ (see Denneberg [1994], chapter four).

\section{$6 \quad$ Second Order stochastic Dominance Equilibria}

In this section, we characterize $\succsim_{2}$ equilibria. We show that the set of $\succsim_{2}$ equilibria coincides with the set of equilibria for strictly averse v.N.M. economy. It also coincides with the set of equilibria for strictly strongly averse utilities. We also show that $\mathrm{a} \succsim_{2}$ equilibrium may be characterized by standard feasibility and budget constraints and the conditions that aggregate endowment is efficient with respect to the price system and its utility price is the sum of individual utility prices. 
Proposition 14 A pair $\left[p^{*},\left(x_{i}^{*}\right)_{i=1}^{n}\right]$ with $\left(x_{i}^{*}\right)_{i=1}^{n}$ feasible is a $\succsim_{2}$ equilibrium iff

1. $p^{*} \gg 0$ and for every $i, E\left(p^{*} x_{i}^{*}\right)=E\left(p^{*} \omega_{i}\right)$.

2. For every $i, x_{i}^{*}$ is a non increasing function of $p^{*}$.

Equivalently $\left[p^{*},\left(x_{i}^{*}\right)_{i=1}^{n}\right]$ fulfills

1. For every $i, E\left(p^{*} x_{i}^{*}\right)=E\left(p^{*} \omega_{i}\right)$,

2. The family of random variables $\left(x_{i}^{*}\right)_{i=1}^{n}$ is comonotone and $\omega$ is a non increasing function of $p^{*}$.

Proof. It follows directly from Proposition 1 and corollary 1.

Proposition 15 A pair $\left[p^{*},\left(x_{i}^{*}\right)_{i=1}^{n}\right]$ is a $\succsim_{2}$ equilibrium iff there exist $\left(u_{i}\right)_{i=1}^{n}$, $u_{i}: \mathbb{R} \rightarrow \mathbb{R}$ strictly concave increasing such that $\left[p^{*},\left(x_{i}^{*}\right)_{i=1}^{n}\right]$ is the equilibrium of the associated v.N.M. economy.

Proof. It follows from corollary 1.

Proposition 16 A pair $\left[p^{*},\left(x_{i}^{*}\right)_{i=1}^{n}\right]$ is an equilibrium of an economy with monotone strictly strongly averse utilities iff it is $a \succsim_{2}$ equilibrium.

Proof. The proof which is obvious is omitted.

We further have:

Proposition 17 A pair $\left[p^{*},\left(x_{i}^{*}\right)_{i=1}^{n}\right]$ with $\left(x_{i}^{*}\right)_{i=1}^{n}$ feasible and $p^{*} \gg 0$ and fully revealing is $a \succsim_{2}$ equilibrium iff

1. For every $i, p^{*} x_{i}^{*}=p^{*} \omega_{i}$,

2. $e\left(p^{*}, \omega\right)=\sum_{i=1}^{n} e\left(p^{*}, x_{i}^{*}\right)$ and $e\left(p^{*}, \omega\right)=E\left(p^{*} \omega\right)$. 
Proof. The proof which is similar to that of Proposition 12 is omitted.

Let $\left(\omega_{i}\right)_{i=1}^{n}$ be fixed. Let $\mathcal{E}_{2}$ be the set of $\succsim_{2}$-equilibria, $\mathcal{E}_{\mathcal{U}}$ be the set of equilibria of a v.N.M. economy with $\left(u_{i}\right)_{i=1}^{n}$ increasing and strictly concave and finally $\mathcal{E}_{\mathcal{V}}$ be the set of equilibria of an economy with monotone $\succ_{2}$ averse utilities. It follows from Propositions 14 and 15 that

$$
\mathcal{E}_{2}=\mathcal{E}_{\mathcal{U}}=\mathcal{E}_{\mathcal{V}}
$$

Other sets of equilibria may be considered: the set of equilibria of a v.N.M. economy with $\left(u_{i}\right)_{i=1}^{n}$ increasing and concave or the set of equilibria of an economy with monotone $\succeq_{2}$ averse utilities. It follows from Lemma 1 and from the proof of Proposition 1 that these sets coincide and are equal to the set of pairs $\left[p^{*},\left(x *_{i}\right)_{i=1}^{n}\right]$ with $p^{*} \gg 0$ and $x_{i}^{*}$ and $p^{*}$ anticomonotone for every $i . w$ and $p^{*}$ are therefore anticomonotone but the family of equilibrium consumptions is not necessarely anticomonotone.

It may also easily be seen that $\mathcal{E}_{2}$ contains the set of CAPM equilibria which are characterized by the property that that consumptions are affine non decreasing functions of the pricing density.

\section{Appendix}

\section{Proof of Lemma 1}

For completeness, we recall the proof which is due to Peleg and Yaari [1975]. Let

$g(x(\ell))=\min \{p(j) ; j$ s.t. $x(j)=x(\ell)\} \quad g^{-}(x(\ell))=\max \{p(j) ; j$ s.t. $x(j)=x(\ell)\}$

Assume w.l.o.g. $x(1) \leq x(2) \leq \cdots x(k)$ Let $g$ be defined as follows :

$$
\begin{aligned}
& g(x)=g(x(1))+(x(1)-x), \quad \text { if } x<x(1) \\
& g(x)=g(x(k)) \exp (x(k)-x), \quad \text { if } x>x(k)
\end{aligned}
$$

and $g$ is right continuous, piecewise linear with potential discontinuity and change of slope at $x(1), x(2), \cdots, x(k)$. Then $g$ is positive and non increasing (decreasing if $x(\ell)<x(j)$ implies $p(\ell)>p(j)$ ). Let $u(x)=\int_{0}^{x} g(t) d t$. Then $u$ is strictly increasing and concave (resp strictly concave if $x(\ell)<x(j)$ implies 
$p(\ell)>p(j))$. Let us remark that $x$ solves $\left\{\begin{array}{l}\max E[u(c)) \text { s.t. } \\ E(p c) \leq E(p x)=w\end{array}\right.$ since the f.o.c., $p(j) \in\left[u_{+}^{\prime}(x(j)), u_{-}^{\prime}(x(j))\right]=\left[g(x(j)), g^{-}(x(j))\right]$ are fulfilled.

\section{Proof of Proposition 1}

Assume that $\xi(p, w) \neq \emptyset$ and let $x \in \xi(p, w)$. If $p(\ell) \leq 0$ for some $\ell$, let $x^{\prime}$ be defined by $x^{\prime}(j)=x(j), \forall j \neq \ell$ and $x^{\prime}(\ell)=x(\ell)+M, M>0$. Then $x^{\prime} \succ_{2} x$ and $E\left(p x^{\prime}\right) \leq w$, a contradiction.

To prove 2, assume that for some pair $(\ell, j), x(\ell)<x(j)$ and $p(\ell) \leq p(j)$.

- Assume $\pi(\ell)<\pi(j)$.

Let $\left\{\begin{array}{l}x^{\prime}(\ell)=x(j) \\ x^{\prime}(j)=x(j)+\frac{\pi(\ell)}{\pi(j)}[x(\ell)-x(j)] \text { and } x^{\prime}(k)=x(k), k \neq(\ell, j) \text {. }\end{array}\right.$

We then have:

$$
\begin{aligned}
E\left(p\left(x^{\prime}-x\right)\right) & =\pi(\ell) p(\ell)\left(x^{\prime}(\ell)-x(\ell)\right)+\pi(j) p(j)\left(\left(x^{\prime}(j)-x(j)\right)\right. \\
& =\pi(\ell) p(\ell)(x(j)-x(\ell))+p(j) \pi(\ell)(x(\ell)-x(j)) \\
& =\pi(\ell)(x(j)-x(\ell))(p(\ell)-p(j)) \leq 0
\end{aligned}
$$

while for any v.N.M. expected utility maximizer with a strictly concave increasing $u: \mathbb{R} \rightarrow \mathbb{R}$

$$
\begin{aligned}
& E\left[u\left(x^{\prime}\right)-u(x)\right] \\
& =\pi(\ell)[u(x(j))-u(x(\ell))]+\pi(j)\left[u\left(x(j)+\frac{\pi(\ell)}{\pi(j)}(x(\ell)-x(j))\right)-u(x(j))\right] \\
& >\pi(\ell)[u(x(j))-u(x(\ell))]+\pi(\ell) u(x(\ell))+[\pi(j)-\pi(\ell)] u(x(j))-\pi(j) u(x(j)) \\
& =0
\end{aligned}
$$

a contradiction.

- If $\pi(j)<\pi(\ell)$, a similar proof may be given with $x^{\prime}(\ell)=x(\ell)+$ $\frac{\pi(j)}{\pi(\ell)}(x(j)-x(\ell)), x^{\prime}(j)=x(\ell)$ and $x^{\prime}(k)=x(k)$ for $k \neq(\ell, j)$. 
- If $\pi(j)=\pi(\ell)$, it follows from the above proof that $x(\ell)<x(j)$ and $p(\ell)>p(j)$ is impossible. Assume that $\pi(j)=\pi(\ell), x(\ell)<x(j)$ and $p(\ell)=p(j)$. Let $x^{\prime}(\ell)=x^{\prime}(j)=\frac{1}{2}(x(\ell)+x(j)), x^{\prime}(k)=x(k), k \neq(l, j)$. Then $E\left(p x^{\prime}\right)=E(p x)$ while $E\left[u\left(x^{\prime}\right)\right]>E[u(x)]$ for any $u$ strictly concave increasing. A contradiction.

Hence $x(\ell)<x(j)$ implies $p(\ell)>p(j)$. In particular $p(\ell)=p(j)$ implies $x(\ell)=x(j)$. Lastly as $x+a \succ_{2} x, \forall a \in \mathbb{R}_{+}^{k}$, if $x \in \xi(p, w)$, then $E(p x)=w$.

Conversely, let $x$ be such that $E(p x)=w$ and $x(\ell)<x(j)$ implies $p(\ell)>$ $p(j)$ and $u$ be given by lemma 1 . Assume that there exists $x^{\prime} \succ_{2} x$ and $E\left(p x^{\prime}\right) \leq$ $w$, then $E\left[u\left(x^{\prime}\right)\right]>E[u(x)]$ while $E\left(p x^{\prime}\right) \leq E(p x)$, contradicting the optimality of $x$, for the v.N.M. utility.

\section{Proof of Proposition 4}

Proof. Clearly if $p$ has a negative coordinate, $e(p, x)=-\infty$. Assume $p \geq 0$. Let us show that $e(p, x) \geq \int_{0}^{1} F_{p}^{-1}(1-t) F_{x}^{-1}(t) d t$. It first follows from HardyLittlewood's theorem that for any $c \in \mathbb{R}^{k}$,

$$
E(p c) \geq \int_{0}^{1}\left[F_{p}^{-1}(1-t) F_{c}^{-1}(t)\right] d t
$$

Assume further that $c \succsim_{2} x$. Since $F_{p}^{-1}(1-t)$ is a non negative, non increasing step function, there exists $\left.\left.a_{i} \in \mathbb{R}_{+}, b_{i} \in\right] 0,1\right]$ such that $F_{p}^{-1}(1-t)=$ $\sum_{i=1}^{k} a_{i} 1_{\left[0, b_{i}\right]}(t)$. Hence

$$
\begin{aligned}
\int_{0}^{1}\left[F_{p}^{-1}(1-t) F_{c}^{-1}(t)\right] d t=\sum_{i=1}^{k} a_{i} \int_{0}^{b_{i}} F_{c}^{-1}(t) d t & \geq \sum_{i=1}^{k} a_{i} \int_{0}^{b_{i}} F_{x}^{-1}(t) d t \\
& =\int_{0}^{1} F_{p}^{-1}(1-t) F_{x}^{-1}(t) d t
\end{aligned}
$$

since $c \succsim_{2} x$.

To prove the converse inequality, it suffices to prove that there exists a contingent claim $c \succsim_{2} x$ such that $E(p c)=\int_{0}^{1} F_{p}^{-1}(1-t) F_{x}^{-1}(t) d t$. Let us relabel states of the world such that $p(1) \geq p(2) \geq \ldots p(k)$. Let $A_{1}, \ldots, A_{k}$ be the partition of $[0,1]$ such that

$$
F_{p}^{-1}(1-t)=p(1) 1_{A_{1}}(t)+\ldots p(l) 1_{A_{l}}(t)+\ldots p(k) 1_{A_{k}}(t)
$$


$A_{1}=[0, \pi(1)], A_{2}=[\pi(1), \pi(1)+\pi(2)], A_{l}=\left[\sum_{i=1}^{l-1} \pi(i), \sum_{i=1}^{l} \pi(i)\right]$. In particular $\lambda\left(A_{l}\right)=\pi(l)$. Let

$$
c(l)=\frac{1}{\pi(l)} \int_{A_{l}} F_{x}^{-1}(t) d t
$$

Since $F_{x}^{-1}$ is non decreasing, $c(1) \leq \ldots \leq c(k)$ and

$$
\sum_{l=1}^{k} p(l) c(l) \pi(l)=\int_{0}^{1} F_{p}^{-1}(1-t) F_{x}^{-1}(t) d t
$$

Consider the contingent claim $c=(c(1), \ldots, c(k))$ in $\mathbb{R}^{k}$. Since $c(1) \leq \ldots \leq$ $c(k), F_{c}^{-1}=c(1) 1_{A_{1}}+\ldots c(l) 1_{A_{l}}+\ldots c(k) 1_{A_{k}}$. By construction, $F_{c}^{-1}=E\left[F_{x}^{-1}\right.$ $\mathcal{C}$ ] where $\mathcal{C}$ is the sigma-field generated by the $\left(A_{i}\right), i=1, \ldots, k$. Hence by Jensen's inequality, $F_{c}^{-1} \succeq_{2} F_{x}^{-1}$. Equivalently $c \succeq_{2} x$

Proof of corollary 2. If $p$ is revealing, the contingent claim $c$ constructed in the previous proof, is the non increasing function of $p f(p)$, with $f$ defined by $f\left(F_{p}^{-1}(1-\mathrm{Id})\right)=E\left(F_{x}^{-1} \mid F_{p}^{-1}(1-\mathrm{Id})\right)$ (the conditionnal expectation being taken with respect to the probability space $([0,1], \mathcal{B}, \lambda)$ with $\lambda$ the Lebesgue measure). Since $p$ is revealing, it follows from proposition 3 that if $c^{\prime} \in \psi(p, x)$, then $c^{\prime}$ is a function of $p$. Let us show that if $c^{\prime}=\phi(p)$ is any (non increasing) function of $p$ that fulfills $c^{\prime} \succsim_{2} x$ and achieves the minimum, then $c=c^{\prime}$. Indeed, let $\left.\left.a_{i}>0, b_{i} \in\right] 0,1\right]$ be such that $F_{p}^{-1}(1-t)=\sum_{i=1}^{k} a_{i} 1_{\left[0, b_{i}\right]}$. We have

$$
\begin{aligned}
\int_{0}^{1}\left[F_{p}^{-1}(1-t) F_{c^{\prime}}^{-1}(t)\right] d t & =\int_{0}^{1}\left[F_{p}^{-1}(1-t) \phi\left(F_{p}^{-1}(1-t)\right) d t\right. \\
& =\sum_{i=1}^{k} a_{i} \int_{0}^{b_{i}} \phi\left(F_{p}^{-1}(1-t)\right) d t \\
& =\sum_{i=1}^{k} a_{i} \int_{0}^{b_{i}} F_{x}^{-1}(t) d t
\end{aligned}
$$

Since $c^{\prime} \succsim_{2} x$, we have $\int_{0}^{b_{i}} \phi\left(F_{p}^{-1}(1-t)\right) d t=\int_{0}^{b_{i}} F_{x}^{-1}(t) d t$ for every $i$, hence $\int_{b_{i}}^{b_{i+1}} \phi\left(F_{p}^{-1}(1-t)\right) d t=\int_{b_{i}}^{b_{i+1}} F_{x}^{-1}(t) d t$. Since $\phi\left(F_{p}^{-1}(1-t)\right)$ is constant on each interval $\left[b_{i}, b_{i+1}\right]$, we get $1_{\left[b_{i}, b_{i+1}\right]}(t) \phi\left(F_{p}^{-1}(1-t)\right)=\frac{1}{\left(b_{i+1}-b_{i}\right)} \int_{b_{i}}^{b_{i+1}} F_{x}^{-1}(t) d t$. Hence $\psi(p, x)$ is a singleton.

The second assertion also follows from the same proof. The function $f$ defined by $f\left(F_{p}^{-1}(1-\mathrm{Id})\right)=E\left(F_{x}^{-1} \mid F_{p}^{-1}(1-\mathrm{Id})\right.$ is that unique function. 


\section{Proof of Proposition 5}

1) Let $r$ be defined by $E(p, 1)=E(p)=\frac{1}{1+r}$ and let $(1+r) e(p, x)=$ $\varphi(x)$. Clearly $\varphi(1)=1$ and $\varphi$ is non decreasing. Furthermore, by Lemma $2, \varphi(x+y)=\varphi(x)+\varphi(y)$ on comonotone functions. Hence by Schmeidler's theorem [1986], $\varphi$ is an integral with respect to a capacity $\nu$. Let us show that $\nu$ is a convex distortion of $P$. Let $x=1_{A}$, then $F_{1_{A}}^{-1}(t)=1_{[1-P(A), 1]}(t)$ and $\nu(A)=\frac{\int_{0}^{P(A)} F_{p}^{-1}(t) d t}{\int_{0}^{1} F_{p}^{-1}(t) d t}=\phi_{p}(P(A))$ where $\phi_{p}(x)=\frac{\int_{0}^{x} F_{p}^{-1}(t) d t}{\int_{0}^{1} F_{p}^{-1}(t) d t}$. Clearly $\phi_{p}$ is a convex distortion. If $x \geq 0,-(1+r) e(p, x)$ is a comonotone coherent measure of risk.

2) The covariance of two anticomonotone variable being non positive, $D(p, x)=$ $\frac{E(x)}{1+r}-e(p, x) \geq 0$. If $x \underset{\tilde{B L}}{\succsim}$, then $F_{y}^{-1}-F_{x}^{-1}$ is non decreasing, hence its covariance with $t \rightarrow F_{p}^{-1}(1-t)$ is non positive. Hence $D(p, y) \geq D(p, x)$. As

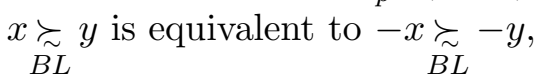

$$
D(p,-y) \geq D(p,-x)
$$

which implies that $\chi(p, x) \leq \chi(p, y)$. One easily verifies that all the other properties of a measure of spread are verified.

\section{Proof of Proposition 6}

To prove assertion 1 , let $x^{\prime} \sim_{2} x$ be such that $x^{\prime}(j)<x^{\prime}(\ell)$ imply $p(j) \geq p(\ell)$. Then there exists $u: \mathbb{R} \rightarrow \mathbb{R}$ concave increasing such that $x^{\prime} \max \left\{\begin{array}{l}E u(c) \text { s.t. } \\ E(p c) \leq E\left(p x^{\prime}\right)\end{array}\right.$. Hence $x^{\prime}$ solves $\left\{\begin{array}{l}\min E(p c) \text { s.t. } \\ E u(c) \geq E u\left(x^{\prime}\right)=E u(x)\end{array}\right.$. If $c \succsim_{2} x$, then $E u(c) \geq E u(x)$, hence $E(p c) \geq E\left(p x^{\prime}\right)$ which proves that $x^{\prime} \in \psi(p, x)$ and that $\psi(p, x)$ contains the convex hull of consumptions $x^{\prime}$ that fulfills $x^{\prime} \sim_{2} x$ and $x^{\prime}(j)<x^{\prime}(\ell)$ implies $p(j) \geq p(\ell)$. Conversely, by Jouini-Kallal's lemma, $\left\{c \succsim_{2} x\right\}=\operatorname{co}\left\{c \sim_{2}\right.$ $x\}+\mathbb{R}_{+}^{k}$, hence if either $c \in \operatorname{co}\left\{c \sim_{2} x\right\}$ and $c$ is not anticomonotone with $p$ or $c>y$ with $y \sim_{2} x$, then $E(p c)>E\left(p x^{\prime}\right)$. Hence $\psi(p, x)$ equals the convex hull of consumptions $x^{\prime}$ that fulfills $x^{\prime} \sim_{2} x$ and $x^{\prime}(j)<x^{\prime}(\ell)$ implies $p(j) \geq p(\ell)$. Assertion 2 follows assertion 1 and proposition 3. Lastly, to prove assertion 3, 
let us first remark that as $\left\{c \mid c \succsim_{2} x\right\} \subseteq\{c \mid E(u(c)) \geq E(u(x))\}$,

$$
\min \left\{\begin{array}{l}
E(p c) \\
c \succsim_{2} x
\end{array} \geq \max _{u} \min _{c}\left\{\begin{array}{l}
E(p c) \\
E u(c) \geq E u(x)
\end{array}\right.\right.
$$

It follows from the proof of assertion 1 that

$$
\min \left\{\begin{array}{l}
E(p c) \\
c \succsim_{2} x
\end{array}=\max _{u} \min _{c}\left\{\begin{array}{l}
E(p c) \\
E u(c) \geq E u(x)
\end{array}=\min _{c}\left\{\begin{array}{l}
E(p c) \\
c \sim_{2} x
\end{array}\right.\right.\right.
$$

\section{Proof of Proposition 8.}

To prove assertion 1 , assume that $x$ is weakly-efficient at price $p$ and that there exists $c \succsim_{2} x$ such that $E(p c)<E(p x)$. Then for $\varepsilon>0, E(u(c+\varepsilon))>E(u(x)$ and fulfills $E(p(c+\varepsilon))<E(p x)$ for $\varepsilon>0$ small enough, contradicting the weak efficiency of $x$. Conversely if $E(p x)=\min \{c \succeq x\} E(p c)$, then by Proposition 3, $x$ and $p$ are anticomonotone and the existence of $u$ follows from Lemma 1 . The equivalence between assertions 1 and 2 follows from Proposition 3 and Lemma 1. The proof of assertion 3 which is similar to that of assertion 1 is omitted.

To prove assertion 4 , for any $x$, we have $E(x)-(1+r) E(p x) \leq(1+$ $r) D(p, x)$. Furthermore $x$ is efficient at price $p$ iff $E(x)-(1+r) E(p x)=(1+$ $r) D(p, x)$. If $E(p x)>0$, as $D(p, x)$ is positively homogeneous, we equivalently have

$$
\frac{E(R(x))-(1+r)}{D(p, R(x))} \leq 1+r, \text { for any } x
$$

where $R(x)=\frac{x}{E(p x)}$ is the return of the contingent claim. There is equality if $x$ is efficient.

\section{Proof of Proposition 10.}

1. implies 2. The original proof is due to Landsberger-Meilijson [1994]. We use a method of proof due to Chateauneuf et al [1997]. Assume that $\left(\bar{x}_{i}\right)_{i=1}^{n}$ are not comonotone. W.l.o.g. we may assume $\bar{x}_{1}(1)>\bar{x}_{1}(2)$ and $\bar{x}_{2}(1)<\bar{x}_{2}(2)$ and $\bar{x}_{1}(1)+\bar{x}_{2}(1) \leq \bar{x}_{1}(2)+\bar{x}_{2}(2)$. Let $x_{1}^{\prime}$ and $x_{2}^{\prime}$ be defined as follows:

$$
\begin{aligned}
& x_{1}^{\prime}(1)=x_{1}^{\prime}(2)=\frac{\pi(1) \bar{x}_{1}(1)+\pi(2) \bar{x}_{1}(2)}{\pi(1)+\pi(2)} \\
& x_{1}^{\prime}(j)=\bar{x}_{1}(j), \forall j>2 .
\end{aligned}
$$


$x_{2}^{\prime}$ is defined so that the feasibility constraint is fulfilled:

$$
\begin{aligned}
x_{2}^{\prime}(1) & =\bar{x}_{2}(1)+\frac{\pi(2)}{\pi(1)+\pi(2)}\left[\bar{x}_{1}(1)-\bar{x}_{1}(2)\right] \\
x_{2}^{\prime}(2) & =\bar{x}_{2}(2)-\frac{\pi(1)}{\pi(1)+\pi(2)}\left[\bar{x}_{1}(1)-\bar{x}_{1}(2)\right] .
\end{aligned}
$$

We have

$$
\begin{aligned}
E\left(x_{i}^{\prime}\right) & =E\left(\bar{x}_{i}\right), i=1,2 \\
\bar{x}_{1}(2)<x_{1}^{\prime}(1) & =x_{1}^{\prime}(2)<\bar{x}_{1}(1) \\
\bar{x}_{2}(1)<x_{2}^{\prime}(1) & \leq x_{2}^{\prime}(2)<\bar{x}_{2}(2) .
\end{aligned}
$$

Hence $x_{1}^{\prime} \succ \bar{x}_{1}$ and $x_{2}^{\prime} \succ \bar{x}_{2}$, a contradiction.

2 implies 3 See Denneberg [1994], chapter four.

3 implies 4. Choose any $p \gg 0$, such that $\omega(j)<\omega(\ell)$ implies $p(j)>p(\ell)$. Then by assertion $3, \bar{x}_{i}(j)<\bar{x}_{i}(\ell)$ implies $\omega(j)<\omega(\ell)$, hence $p(j)>p(\ell)$. By lemma 1 , there exists, $u_{i}: \mathbb{R} \rightarrow \mathbb{R}$ increasing strictly concave such that $\bar{x}_{i}$ solves $\left\{\begin{array}{l}\max E u_{i}(c) \text { s.t. } \\ E(p c) \leq E\left(p \bar{x}_{i}\right)\end{array}\right.$ The pair $\left[p,\left(\bar{x}_{i}\right)_{i=1}^{n}\right]$ is therefore an equilibrium with transfer payments of the associated v.N.M. economy.

4 implies 5. Let $\left(u_{i}\right)_{i=1}^{n}$ be as constructed in 4 . Then $\left(\bar{x}_{i}\right)_{i=1}^{n}$ is a Pareto optimum of the associated v.N.M. economy.

5 implies 1 The proof which is obvious is omitted

\section{Proof of Proposition 11.}

1 implies 2. If $\left(\bar{x}_{i}\right)_{i=1}^{n}$ is a $\succsim_{2}$ Pareto optimum, then from Proposition 10 assertion 4 , for any $p \gg 0$ such that $\omega(j)<\omega(\ell)$ implies $p(j)>p(\ell),\left[p,\left(\bar{x}_{i}\right)_{i=1}^{n}\right]$ is an equilibrium with transfer payments of a strictly concave economy, hence by Proposition $9, \mathrm{a} \succsim_{2}$ equilibrium with transfer payments.

2 implies 1 . Let $\left[p,\left(\bar{x}_{i}\right)_{i=1}^{n}\right]$ be a $\succsim_{2}$ equilibrium with transfer payments with $p \gg 0$. Assume that $\left.\left(\bar{x}_{i}\right)_{i=1}^{n}\right)$ is not $\succsim_{2}$ Pareto optimal. Then there exists a

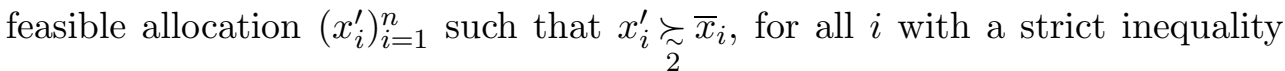
for some $i$. If $x_{i}^{\prime} \neq \bar{x}_{i}$, by convexity, we may assume that $x_{i}^{\prime} \succ_{2} \bar{x}_{i}$. Hence $E\left(p x_{i}^{\prime}\right)>E\left(p \bar{x}_{i}\right)$, for all $i$ such that $x_{i}^{\prime} \neq \bar{x}_{i}$. Summing over $i$, we get a contradiction with the feasibility of $\left(x_{i}^{\prime}\right)_{i=1}^{n}$. Hence $\left.\left(\bar{x}_{i}\right)_{i=1}^{n}\right)$ is a $\succsim_{2}$ Pareto optimum. 


\section{References}

Atkinson A.B. 1970. On the measurement of Inequality, Journal of Economic Theory, 2, pp.244-263.

Atzner P., Delbaen F, Eber J.M. and Heath D. 1999. Coherent Measures of Risk, Mathematical Finance, 9-3, pp.203-228.

Aubin J. P. 1998. Optima and Equilibria, An Introduction to non Linear Analysis, Second edition, Springer, Graduate texts in mathematics.

Bickel P.J. and Lehmann E. L. 1976. Descriptive statistics for non-parametric models, III. Dispersion, Annals of Statistics, 4, pp.1139-1158.

Bickel P.J. and Lehmann E. L. 1979. Descriptive statistics for non-parametric models, IV. Spread, in Contributions to statistics, Jureckova (Ed.), Reidel.

Chateauneuf A., Cohen M. and Kast R. 1997. Comonotone random variables in Economics, A review of some results, Working Paper, Cermcem.

Chateauneuf A., Cohen M. and Meilijson I. 1997. New tools to better model behavior under risk and uncertainty : An overview, Finance, 18, pp25-46.

Chew S.H. and Mao M.H. 1995. A Schur Concave Characterization of Risk Aversion for Non Expected Utility Preferences, Journal of Economic Theory, 67, pp.402-435.

Chew S.H. and Zilcha I. 1990, Invariance of the efficient sets when the expected utility hypothesis is relaxed, Journal of Economic Behaviour and Organisation, 13, pp.125-131.

Delbaen F. 2000. Coherent Measures of Risk on General Probability Space, Preprint.

Denneberg D. 1994. Non-additive Measures and Integral, Kluwer Academic Publishers, Holland.

Denneberg D. 1990. Why standard deviation should be replaced by absolute deviation, Astin Bulletin, 20. 
Dybvig, P. 1988. Distributionnal Analysis of Portfolio Choice, Journal of Business, 61, pp.369-393.

Dybvig, P. and Ross S. 1982. Portfolio Efficient Sets, Econometrica, 50, pp.1525-1546.

Föllmer H. and Schied A. 2002. Stochastic finance. An introduction in discrete time. De Gruyter editor, Berlin.

Gollier C. and Schlesinger, H 1996. Arrow's theorem on the optimality of deductibles: A stochastic dominance approach, Economic Theory, 22, pp.107-110.

Jouini C. and Kallal H. 2000. Efficient Trading Strategies in the Presence of Market Frictions, Review of Financial Studies, 14, pp.343-369.

Kim C. 1998. Stochastic dominance, Pareto Optimality and Equilibrium Pricing, Review of Economic Studies, 65, pp.341-356.

Landsberger M. and Meilijson 1994. Comonotone allocations, Bickel Lehmann dispersion and the Arrow-Pratt measure of risk aversion, Annals of Operation Research, 52, pp.97-106.

Peleg B. and Yaari, M.E. 1975. A Price Characterisation of Efficient Random Variables, Econometrica, 43, pp.283-292.

Rothschild M. and Stiglitz, J.E. 1970. Increasing Risk, I, A Definition, Journal of Economic Theory, 2, pp.225-243.

Schmeidler D. 1986. Integral Representation Without Additivity, Proceedings of the American Mathematical Society, 97, 2, 255-261 